\title{
Biochemical Markers as Predictors of In-Hospital Mortality in Patients with Severe Trauma: A Retrospective Cohort Study
}

\author{
Ha Nee Jang', Hyun Oh Park², Tae Won Yang ${ }^{3}$, Jun Ho Yang', Sung Hwan Kim², Seong Ho Moon ${ }^{2}$, \\ Joung Hun Byun ${ }^{2}$, Chung Eun Lee ${ }^{4}$ Jong Woo Kim² ${ }^{2}$ Dong Hun Kang ${ }^{4}$, and Kyeong Hee Baek ${ }^{5}$ \\ ${ }^{1}$ Department of Internal Medicine, Gyeongsang National University Hospital, Gyeongsang National University School of Medicine and Institute of Health Sciences, Jinju; \\ 2Department of Thoracic and Cardiovascular Surgery, Gyeongsang National University Changwon Hospital, Gyeongsang National University School of Medicine and Institute of Health \\ Sciences, Changwon; ${ }^{3}$ Department of Neurology, Gyeongsang National University Changwon Hospital, Changwon; ${ }^{4}$ Department of Thoracic and Cardiovascular Surgery, Gyeongsang \\ National University Hospital, Gyeongsang National University School of Medicine and Institute of Health Sciences, Jinju; \\ ${ }^{5}$ Department of Neurosurgery, Gyeongsang National University Changwon Hospital, Changwon, Korea
}

Background: Initial evaluation of injury severity in trauma patients is an important and challenging task. We aimed to assess whether easily measurable biochemical parameters (hemoglobin, $\mathrm{pH}$, and prothrombin time/international normalized ratio [PT/INR]) can predict in-hospital mortality in patients with severe trauma.

Methods: This retrospective study involved review of the medical records of 315 patients with severe trauma and an injury severity score $>15$ who were managed at Gyeongsang National University Hospital between January 2005 and December 2015. We extracted the following data: in-hospital mortality, injury severity score, and initial hemoglobin level, $\mathrm{pH}$, and PT/INR. The predictive values of these variables were compared using receiver operation characteristic curves.

Results: Of the 315 patients, 72 (22.9\%) died. The in-hospital mortality rates of patients with hemoglobin levels $<8.4 \mathrm{~g} / \mathrm{dl}$ and $\geq 8.4$ $\mathrm{g} / \mathrm{dl}$ were $49.8 \%$ and $9.9 \%$, respectively $(P<0.001)$. At a cutoff hemoglobin level of $8.4 \mathrm{~g} / \mathrm{dl}$, the sensitivity and specificity values for mortality were $81.9 \%$ and $86.4 \%$, respectively. At a pH cutoff of 7.25 , the sensitivity and specificity values for mortality were $66.7 \%$ and $77.8 \%$, respectively; $66.7 \%$ of patients with a $\mathrm{pH}<7.25$ died versus $22.2 \%$ with a $\mathrm{pH} \geq 7.25(\mathrm{P}<0.001)$. The in-hospital mortality rates for patients with PT/INR values $\geq 1.4$ and $<1.4$ were $37.5 \%$ and $16 \%$, respectively $(P<0.001$; sensitivity, $37.5 \%$; specificity, $84 \%)$. Conclusions: Using the suggested cutoff values, hemoglobin level, $\mathrm{pH}$, and PT/INR can simply and easily be used to predict in-hospital mortality in patients with severe trauma.

Key Words: acidosis; hemoglobin; injuries; international normalized ratio; mortality.

The ability to predict injury severity quickly and accurately should lead to improved patient outcomes. Patient treatment and disposition (intensive care unit or ward) is determined by these initial assessments [1]. Several trauma scores have been developed to predict injury severity and the risk of mortality; the injury severity score (ISS) is the most commonly used. The ISS correlates with mortality, and severe trauma is defined as an ISS $>15$ [2]. Despite being commonly

\footnotetext{
Received on June 13, 2017 Revised on July 27, 2017 Accepted on August 1, 2017

Correspondence to: Hyun Oh Park, Department of Thoracic and Cardiovascular Surgery, Gyeongsang National University Changwon Hospital, Gyeongsang National University School of Medicine and Institute of Health Sciences, 11 Samjeongja-ro, Seongsan-gu, Changwon 51472, Korea

Tel: +82-55-214-3849, Fax: +82-55-214-3260, E-mail: romejuliet@naver.com
}

*No potential conflict of interest relevant to this article was reported.

(c) This is an Open Access article distributed under the terms of the Creative Commons Attribution Non-Commercial License (http://creativecommons.org/ licenses/by-nc/4.0/) which permits unrestricted non-commercial use, distribution, and reproduction in any medium, provided the original work is properly cited. Copyright (c) 2017 The Korean Society of Critical Care Medicine 
used to predict mortality, there are limitations to using the ISS as a decision-making tool in the clinical setting. It is complex and time-consuming to calculate, and is therefore generally used for audit and research purposes rather than for clinical decision making [1]. To counter this disadvantage, several studies have attempted to identify biochemical markers that can be used to predict mortality $[1,3]$.

The trauma triad for death in patients with severe traumatic injuries comprises hypothermia, acidosis, and coagulopathy. Severe hemorrhage in trauma reduces oxygen delivery and may lead to hypothermia, acidosis, and coagulopathy [3]. In 1982, Kashuk et al. [4] showed that a "bloody vicious cycle" involving hemorrhage and tissue injury in patients with severe trauma causes this predictable triad of complicating factors. They suggested that medical providers have an accurate understanding of the triad and that this understanding should serve as the cornerstone of all interventions provided to patients who have sustained severe traumatic injury. Based on this triad, we hypothesized that hemoglobin level (HbL), potential of hydrogen level (pHL), and prothrombin time/ international normalized ratio (PT/INR) on arrival would be early predictors of mortality. We attempted to analyze the usefulness of these biochemical values as predictors of in-hospital mortality in severe trauma patients with an ISS $>15$, and analyzed the correlations between these values and the ISS.

\section{Materials and Methods}

\section{1) Study population}

We retrospectively and consecutively evaluated all patients with an ISS $>15$ who were treated for severe trauma between January 2005 and December 2015 at Gyeongsang National University Hospital. We identified 454 eligible patients. We excluded patients who had penetrating injuries, those who were discharged from the emergency department, and those who received fluid in the field. Based on these criteria, 139 patients were

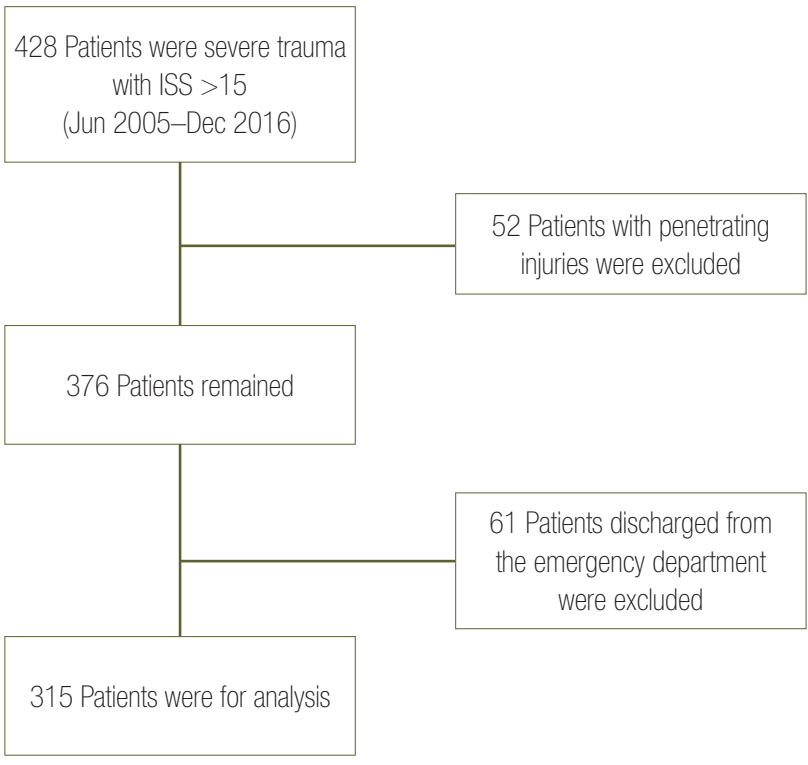

Figure 1. Outline of patient selection and exclusion. ISS: injury severity score.

excluded and 315 patients were finally included in our study (Figure 1). The medical records and electronic laboratory results were reviewed and data were extracted on demographics; mechanism of injury; ISS; HbL, pHL, and PT/INR on arrival at the emergency department; and in-hospital mortality.

\section{2) Treatment protocol}

Since 2008, the center has provided a 24-hour service. During each shift, an emergency medicine specialistwho acts as the trauma team's leader-resides in the hospital for the prompt treatment of trauma patients. Patients are classified as being "severe trauma patients" if they are expected, on initial examination by an emergency medicine specialist, to have an ISS $>15$. Patients classified as having severe trauma are examined within 1 hour, in cooperation with related departments, and most are admitted to the intensive care unit.

\section{3) Definitions}

The ISS is an anatomic scoring system that provides an overall score for patients with multiple injuries. Each injury is assigned an abbreviated injury scale score and is allocated to 1 of the following six body regions: head, 
face, chest, abdomen, extremities (including the pelvis), and external. Only the highest abbreviated injury scale score for each body region is used. The three most severely injured body regions have their scores squared and summed to produce the ISS score. Severe trauma is defined as an ISS $>15$ [2]. Cutoff values were estimated by using receiver operating characteristic (ROC) curves. The highest value of the sum of sensitivity and specificity was defined as the cutoff value. Mortality was defined as all-cause in-hospital mortality; we did not evaluate specific causes of death.

\section{4) Data analysis}

Missing data were not replaced or imputed. We calculated P-values using Pearson chi-square test or Fisher exact for categorical variables, and continuous data were correlated using the Pearson correlation coefficient. A Pvalue $<0.05$ was considered statistically significant. The predictive values of these variables were determined using ROC curves, with Bonferroni corrections performed

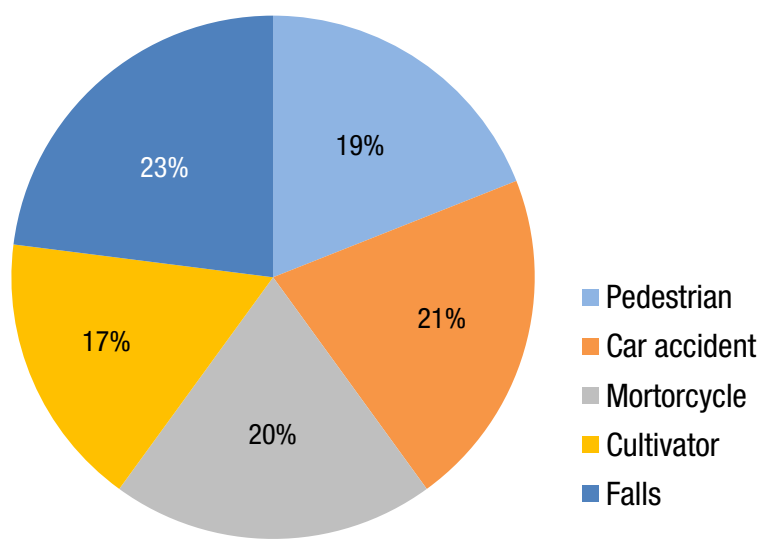

Figure 2. Mechanism of injury. for multiple comparisons. All statistical analyses were performed using SPSS version 24.0 (IBM Corp., Armonk, NY, USA) and R version 3.3.4 for Windows (R Foundation for Statistical Computing, Vienna, Austria).

\section{5) Ethics approval}

This retrospective medical record review of the trauma registry at Gyeongsang National University Hospital was approved by Institutional Review Board (No. GNUH 2017-06-024).

\section{Results}

Between January 2005 and December 2015, our hospital managed 315 patients with severe trauma. Of these, 255 patients $(81 \%)$ were male, $60(19 \%)$ were female, and $72(22.9 \%)$ died. Motor vehicle collisions were main cause of trauma (77\%): these included car (21\%), motorcycle $(20 \%)$, pedestrian $(19 \%)$, and cultivator $(17 \%)$ accidents (Figure 2). The age, HbL, pHL, PT/INR, and ISS distributions are shown in Table 1.

ROC curves were used to estimate the sensitivity, specificity, and cutoff values of $\mathrm{HbL}, \mathrm{pHL}$, and PT/INR to predict in-hospital mortality. The in-hospital mortality rate for severely injured trauma patients with an $\mathrm{HbL}$ $<8.4 \mathrm{~g} / \mathrm{dl}$ was $49.8 \%$, compared with an in-hospital mortality rate of $9.9 \%$ for those with an $\mathrm{HbL} \geq 8.4 \mathrm{~g} / \mathrm{dl}$ (P $<0.001$; odds ratio [OR], 13.56). At this level, the $\mathrm{HbL}$ had a sensitivity of $81.9 \%$ and a specificity of $86.4 \%$ for mortality. Hence, an $\mathrm{HbL}$ of $8.4 \mathrm{~g} / \mathrm{dl}$ was determined to be the cutoff value for in-hospital mortality. The posi-

Table 1. Distribution of age, hemoglobin, potential of hydrogen, INR, and ISS

\begin{tabular}{lcccc}
\hline Variable & Median & Minimum & Maximum & Interquartile range \\
\hline Age $(\mathrm{yr})$ & 66 & 14 & 87 & $51-74$ \\
$\mathrm{Hb}(\mathrm{g} / \mathrm{dl})$ & 11.9 & 5.4 & 19.7 & $9.0-14.0$ \\
$\mathrm{PH}$ & 7.37 & 6.89 & 7.55 & $7.25-7.42$ \\
$\mathrm{PT} / \mathrm{NRR}$ & 1.22 & 0.90 & 2.85 & $1.11-1.38$ \\
$\mathrm{ISS}$ & 36 & 17 & 59 & $33-43$ \\
\hline
\end{tabular}

INR: international normalized ratio; ISS: injury severity score; Hb: hemoglobin; PT: prothrombin time. 
Table 2. Pairwise comparison of the AUC values

\begin{tabular}{lcccc}
\hline Variable & Difference between AUC values & Standard error & Z & P-value \\
\hline Hb-pH & 0.161 & 0.041 & 3.898 & $<0.001$ \\
HbL-PT/INR & 0.302 & 0.043 & 7.084 & $<0.001$ \\
Hb-ISS & 0.266 & 0.027 & 6.487 & $<0.001$ \\
pH-PT/INR & 0.143 & 0.143 & 2.683 & 0.044 \\
pH-ISS & 0.107 & 0.107 & 2.059 & 0.237 \\
\hline PT/INR-ISS & 0.036 & 0.036 & 0.672 & 1 \\
\hline
\end{tabular}

AUC: area under the curve; Hb: hemoglobin; HbL: hemoglobin level; PT: prothrombin time; INR: international normalized ratio; ISS: injury severity score.

Table 3. The sensitivity, specificity, PPV, and NPV for in-hospital mortality

\begin{tabular}{lcccc}
\hline Variable & $\mathrm{Hb}<8.4 \mathrm{~g} / \mathrm{dl}$ & $\mathrm{pH}<7.25$ & $\mathrm{PT} / \mathrm{INR} \geq 1.4$ & Composite group $^{\mathrm{a}}$ \\
\hline Mortality (\%) & 49.8 & 66.7 & 37.5 & 87.5 \\
Sensitivity (\%) & 81.9 & 66.7 & 37.5 & 36.8 \\
Specificity (\%) & 86.4 & 77.8 & 84.0 & 97.1 \\
PPV (\%) & 59.8 & 47.1 & 40.9 & 87.5 \\
NPV (\%) & 90.1 & 88.7 & 81.9 & 73.9 \\
\hline
\end{tabular}

PPV: positive predictive value; NPV: negative predictive value; Hb: hemoglobin; PT: prothrombin time; INR: international normalized ratio.

aPatients with an hemoglobin level $<8.4 \mathrm{~g} / \mathrm{dl}$, potential of hydrogen level $<7.25$, and PT/INR level $\geq 1.4$.

tive predictive value (PPV) was lower than expected at $59.8 \%$, but the negative predictive value (NPV) was higher than expected at $90.1 \%$. A pHL of 7.25 was evaluated as the cutoff value for in-hospital mortality, as $66.7 \%$ of patients with a $\mathrm{pHL}<7.25$ died versus $22.2 \%$ with a $\mathrm{pHL} \geq 7.25(\mathrm{P}<0.001 ; \mathrm{OR}, 7.0)$. At this cutoff value, the sensitivity and specificity values were $66.7 \%$ and $77.8 \%$, respectively. The PPV was once again low at $47.1 \%$, and the NPV was high at $88.7 \%$. A PT/INR value of 1.4 was estimated as being the cutoff for in-hospital mortality, as $37.5 \%$ of patients with a PT/INR value $\geq 1.4$ died versus $16 \%$ with a $\mathrm{PT} / \mathrm{INR}$ value $<1.4$ ( $\mathrm{P}<$ $0.001 ;$ OR, 3.14). At this cutoff value, the sensitivity was $37.5 \%$, specificity $84 \%$, PPV $40.9 \%$, and NPV $81.9 \%$.

A low $\mathrm{HbL}$ was considerably more specific than a low pHL or elevated PT/INR for predicting in-hospital mortality (Table 2). Combining these three values in an analysis yielded the following results: $87.5 \%$ of patients with an $\mathrm{HbL}<8.4$, pHL $<7.25$, and PT/INR level $\geq 1.4$ died. In contrast, $35.2 \%$ of patients who met only one or two of these three cutoff values died. This composite had a sensitivity of $36.8 \%$ and a specificity of $97.1 \%$ for mortality; the PPV was $87.5 \%$ and the NPV $73.9 \%$ (Table 3).

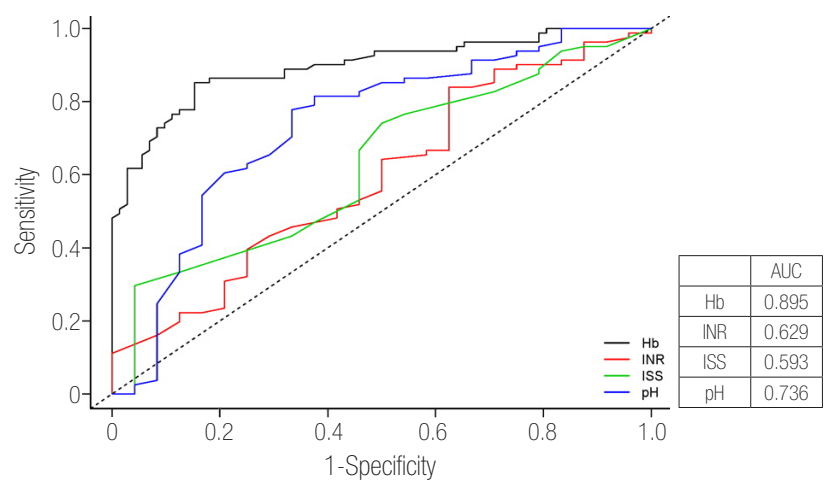

Figure 3. Receiver operation characteristic curves for in-hospital mortality. AUC, area under the curve; Hb: hemoglobin; INR, international normalized ratio; ISS, injury severity score.

The ROC was used to demonstrate the sensitivity and specificity of the HbL, pHL, PT/INR, and ISS for predicting in-hospital mortality (Figure 3). The HbL (area under the curve [AUC], 0.895) was a more significant biochemical predictor of in-hospital mortality than $\mathrm{pHL}$ (AUC, 0.736) and PT/INR (AUC, 0.593). Using Pearson correlation coefficients (Table 4), the ISS correlated significantly with $\mathrm{HbL}, \mathrm{pHL}$, and PT/INR $(\mathrm{P}=0.01)$. 
Table 4. Pearson correlation coefficients

\begin{tabular}{|c|c|c|c|c|}
\hline Variable & $\mathrm{Hb}$ & $\mathrm{pH}$ & PT/INR & ISS \\
\hline \multicolumn{5}{|l|}{$\mathrm{Hb}$} \\
\hline Pearson correlation & 1 & $0.228^{\mathrm{a}}$ & $-0.154^{\mathrm{a}}$ & $-0.151^{\mathrm{a}}$ \\
\hline P-value (two-tailed) & & $<0.001$ & 0.006 & 0.007 \\
\hline \multicolumn{5}{|l|}{$\mathrm{pH}$} \\
\hline Pearson correlation & $0.228^{\mathrm{a}}$ & 1 & $-0.202^{\mathrm{a}}$ & $-0.345^{\mathrm{a}}$ \\
\hline P-value (two-tailed) & $<0.001$ & & $<0.001$ & $<0.001$ \\
\hline \multicolumn{5}{|l|}{$\mathrm{PT} / \mathrm{NRR}$} \\
\hline Pearson correlation & $-0.154^{\mathrm{a}}$ & $-0.202^{\mathrm{a}}$ & 1 & $0.223^{\mathrm{a}}$ \\
\hline P-value (two-tailed) & 0.006 & $<0.001$ & & $<0.001$ \\
\hline \multicolumn{5}{|l|}{ ISS } \\
\hline Pearson correlation & $-0.151^{\mathrm{a}}$ & $-0.345^{\mathrm{a}}$ & $0.223^{\mathrm{a}}$ & 1 \\
\hline P-value (two-tailed) & 0.007 & $<0.001$ & $<0.001$ & \\
\hline
\end{tabular}

Hb: hemoglobin; PT: prothrombin time; INR: international normalized ratio; ISS: injury severity score.

${ }^{a}$ Correlation is significant at the level $\mathrm{P}<0.01$ (two-tailed).

\section{Discussion}

Several trauma scores have proven useful as indicators of mortality. These can be used to identify the severity of trauma injury and might influence therapeutic decisions. The ISS is one of these scores. Although numerous recent publications have questioned its accuracy, it remains the most commonly used trauma score internationally $[5,6]$. The ISS can be used to identify patients with severe traumatic injury, but it requires knowledge of all anatomical injuries [7]. Complete diagnosis of all injuries may take many hours after admission to the emergency department, thus, the usefulness of the score in clinical decision making is limited [1]. Because the ISS has such limitations, we hypothesized that $\mathrm{HbL}, \mathrm{pHL}$, and PT/INR value would be early predictors of mortality, based on the trauma triad of death $[3,4]$. We analyzed the correlations between in-hospital mortality and HbL, pHL, PT/INR, and ISS using ROC curves; all values were statistically significant (AUC: HbL, 0.895; pHL, 0.736; PT/INR, 0.593; and ISS, 0.629). Pearson correlation coefficients showed that the ISS correlated significantly with $\mathrm{HbL}$, $\mathrm{pHL}$, and PT/INR level $(\mathrm{P}=0.01)$.

Knottenbelt [8] suggested that the initial hemoglobin measurement may prove useful as a predictor of mortality in patients with severe traumatic injury. In that study, the initial HbLs of 1,000 patients were collected and analyzed; the mortality rate was higher in those with an $\mathrm{HbL}$ $<8 \mathrm{~g} / \mathrm{dl}$ than with an $\mathrm{HbL} \geq 8 \mathrm{~g} / \mathrm{dl}(\mathrm{P}<0.001)$. Knottenbelt [8] thus suggested that a low HbL observed soon after injury is usually an indicator of serious ongoing hemorrhage and has important implications for management and prognosis. Another study showed that hemorrhage in trauma patients is associated with an early decrease in $\mathrm{HbL}$. An $\mathrm{HbL} \leq 10 \mathrm{~g} / \mathrm{dl}$ within the first 30 minutes of patient arrival will correctly identify the presence or absence of significant bleeding. Based on that finding, the authors suggested that a hemoglobin drop within minutes of sustaining injuries can predict mortality [9]. Similarly, we found that an $\mathrm{HbL}<8.4 \mathrm{~g} / \mathrm{dl}$ predicted in-hospital mortality in patients with severe trauma (AUC, 0.895; P $<0.001)$.

Acidosis can induce organ dysfunction, affecting the heart, kidneys, and liver and can increase pulmonary vascular resistance. This may result in hypoperfusion [10]. The utility of $\mathrm{pH}$ in the assessment of trauma patients has been debated in the literature [10-12]. Kaplan and Kellum [10] showed that the initial $\mathrm{pH}$ measured in the emergency department discriminated survivors from non- 
survivors of major vascular injury. Other studies showed that acidemia was correlated with serum lactate-lactate levels were higher in patients with acidemia - and that in patients with severe trauma, metabolic acidosis was correlated with survival $[11,12]$. Those research findings are similar to ours. We found that academia $(\mathrm{pH}<7.25)$ on arrival can be used to predict in-hospital mortality, as it was correlated with in-hospital mortality in patients with severe traumatic injury (AUC, 0.736; $\mathrm{P}<0.001$ ).

Traumatic coagulopathy is a hypocoagulable state that occurs in most patients with severe trauma. Immediately after sustaining severe injury, hypoperfusion may induce coagulopathy. This coagulopathy is caused by increased anticoagulation and hyperfibrinolysis via increased production of protein $\mathrm{C}$ and tissue plasminogen activators and decreased concentrations of plasminogen activator inhibitors and thrombin activatable fibrinolysis inhibitors [13-15]. PT/INR is commonly measured in trauma patients; it is used as a measure of coagulopathy. Verma and Kole [16] published a study about the association between PT/INR and mortality in trauma patients. They analyzed 99 trauma patients and showed that the INR is a good predictor of mortality and has high diagnostic accuracy. Peltan et al. [17] also showed that an INR-based definition of acute traumatic coagulopathy is associated with mortality. In their multicenter prospective observational study, acute traumatic coagulopathy (defined as a PT/INR $>1.5$ ) was significantly associated with allcause mortality $(\mathrm{OR}, 1.88 ; \mathrm{P}<0.001)$ [17]. In our study, the PT/INR cutoff value was $\geq 1.4$, but this variable had relatively low statistical significance (AUC, 0.593; P < $0.001)$.

Our study has several limitations. First, we only evaluated trauma patients managed at a single hospital, which may have introduced selection bias and limits the extrapolation of our findings to the entire population. Second, the sample size was relatively small; larger studies are needed to validate our findings regarding the predictors of mortality in patients with severe trauma. Third, our study was a retrospective evaluation, and as with all trauma registries, the accuracy of the recorded data may vary [18]. However, it should be noted that while the ISS can be a subjective measure, the HbL, pHL, and PT/ INR are objective measures. Moreover, the $\mathrm{HbL}, \mathrm{pHL}$, and PT/INR values were recorded more accurately than were the ISS scores. While this weakens the comparison between the variables, it strengthens the argument for the use of biochemical scores to predict mortality. Last, we excluded some patients from the study because they had received fluids in the field. We excluded these patients to prevent skewing the results based on presumed hemodilution or the hyperchloremic acidosis that may be caused by rapid saline infusion [19].

Despite these limitations, this study is an important investigation of predictors associated with in-hospital mortality in patients with severe traumatic injury. Our study showed that initial HbL, pHL, and PT/INR were significant predictors of in-hospital mortality in severely injured trauma patients. Comparisons of these findings with those of other reports will enhance the prediction of mortality. We anticipate that these biochemical predictors of in-hospital mortality can more easily be used in an emergency department setting. Our findings suggest that close monitoring should be considered for patients with severe trauma and an $\mathrm{HbL}<8.4$, $\mathrm{pHL}<7.25$, or $\mathrm{PT} / \mathrm{INR} \geq 1.4$.

\section{ORCID}

$\begin{array}{ll}\text { Ha Nee Jang } & \text { http://orcid.org/0000-0002-1402-931x } \\ \text { Hyun Oh Park } & \text { http://orcid.org/0000-0003-1302-6456 } \\ \text { Tae Won Yang } & \text { http://orcid.org/0000-0002-8113-2384 } \\ \text { Jun Ho Yang } & \text { http://orcid.org/0000-0002-7508-5307 } \\ \text { Sung Hwan Kim } & \text { http://orcid.org/0000-0002-6888-774X } \\ \text { Seong Ho Moon } & \text { http://orcid.org/0000-0001-8043-1588 } \\ \text { Joung Hun Byun } & \text { http://orcid.org/0000-0003-1909-664x } \\ \text { Chung Eun Lee } & \text { http://orcid.org/0000-0003-4469-7201 } \\ \text { Jong Woo Kim } & \text { http://orcid.org/0000-0003-2578-9821 } \\ \text { Dong Hun Kang } & \text { http://orcid.org/0000-0003-3012-4197 } \\ \text { Kyeong Hee Baek } & \text { http://orcid.org/0000-0003-4100-107x }\end{array}$




\section{References}

1. Sammour T, Kahokehr A, Caldwell S, Hill AG. Venous glucose and arterial lactate as biochemical predictors of mortality in clinically severely injured trauma patients: a comparison with ISS and TRISS. Injury 2009;40:104-8.

2. Copes WS, Champion HR, Sacco WJ, Lawnick MM, Keast SL, Bain LW. The Injury Severity Score revisited. J Trauma 1988;28:69-77.

3. Mikhail J. The trauma triad of death: hypothermia, acidosis, and coagulopathy. AACN Clin Issues 1999;10:85-94.

4. Kashuk JL, Moore EE, Millikan JS, Moore JB. Major abdominal vascular trauma: a unified approach. J Trauma 1982;22:672-9.

5. Lavoie A, Moore L, LeSage N, Liberman M, Sampalis JS. The new Injury Severity Score: a more accurate predictor of in-hospital mortality than the Injury Severity Score. J Trauma 2004;56:1312-20.

6. Rutledge R, Fakhry S, Rutherford E, Muakkassa F, Meyer A. Comparison of APACHE II, Trauma Score, and Injury Severity Score as predictors of outcome in critically injured trauma patients. Am J Surg 1993;166:244-7.

7. Raum MR, Nijsten MW, Vogelzang M, Schuring F, Lefering R, Bouillon B, et al. Emergency trauma score: an instrument for early estimation of trauma severity. Crit Care Med 2009;37:1972-7.

8. Knottenbelt JD. Low initial hemoglobin levels in trauma patients: an important indicator of ongoing hemorrhage. J Trauma 1991;31:1396-9.

9. Bruns B, Lindsey M, Rowe K, Brown S, Minei JP, Gentilello LM, et al. Hemoglobin drops within minutes of injuries and predicts need for an intervention to stop hemorrhage. J Trauma 2007;63:312-5.
10. Kaplan LJ, Kellum JA. Initial pH, base deficit, lactate, anion gap, strong ion difference, and strong ion gap predict outcome from major vascular injury. Crit Care Med 2004;32:1120-4.

11. Summersgill A, Kanter M, Fraser RM, Caputo ND, Simon R. Determining the utility of metabolic acidosis for trauma patients in the emergency department. J Emerg Med 2015;48:693-8.

12. Mizock BA, Falk JL. Lactic acidosis in critical illness. Crit Care Med 1992;20:80-93.

13. Spinella PC, Holcomb JB. Resuscitation and transfusion principles for traumatic hemorrhagic shock. Blood Rev 2009;23:231-40.

14. Brohi K, Cohen MJ, Ganter MT, Schultz MJ, Levi M, Mackersie RC, et al. Acute coagulopathy of trauma: hypoperfusion induces systemic anticoagulation and hyperfibrinolysis. J Trauma 2008;6:1211-7.

15. Brohi K. Trauma induced coagulopathy. J R Army Med Corps 2009;155:320-2.

16. Verma A, Kole T. International normalized ratio as a predictor of mortality in trauma patients in India. World J Emerg Med 2014;5:192-5.

17. Peltan ID, Vande Vusse LK, Maier RV, Watkins TR. An international normalized ratio-based definition of acute traumatic coagulopathy is associated with mortality, venous thromboembolism, and multiple organ failure after injury. Crit Care Med 2015;43:1429-38.

18. Owen JL, Bolenbaucher RM, Moore ML. Trauma registry databases: a comparison of data abstraction, interpretation, and entry at two level I trauma centers. J Trauma 1999;46:1100-4.

19. Scheingraber S, Rehm M, Sehmisch C, Finsterer U. Rapid saline infusion produces hyperchloremic acidosis in patients undergoing gynecologic surgery. Anesthesiology 1999;90:1265-70. 